\title{
Comparative Study on the Difference in Functional Outcomes at Discharge between Proximal and Total Gastrectomy
}

\author{
Kazuaki Kuwabara $^{a}$ Shinya Matsuda ${ }^{b} \quad$ Kiyohide Fushimi $^{c}$ \\ Koichi B. Ishikawa ${ }^{d}$ Hiromasa Horiguchi $^{\text {e }}$ Kenji Fujimori $^{f}$ \\ ${ }^{a}$ Department of Health Care Administration and Management, Graduate School \\ of Medical Sciences, Kyushu University, Fukuoka, ${ }^{b}$ Department of Preventive \\ Medicine and Community Health, University of Occupational and \\ Environmental Health, Kitakyushu, 'Department of Health Policy and \\ Informatics, Tokyo Medical and Dental University, Tokyo, 'Economics Section, \\ Surveillance Division, Center for Cancer Control and Information Services, \\ National Cancer Center, Tokyo, ${ }^{\mathrm{e}}$ Department of Health Management and \\ Policy, Graduate School of Medicine, Tokyo University, Tokyo, and ${ }^{f}$ Center for \\ Regional Healthcare and Certified Educator Support, Hokkaido University, \\ Sapporo, Japan
}

\section{Key Words}

Complications - Distal gastrectomy - Functional outcome · Proximal gastrectomy · Total gastrectomy

\begin{abstract}
Several studies have regarded proximal gastrectomy (PG) as optimal compared to total gastrectomy (TG) for upper stomach cancer. In addition to the traditional outcomes of complication and mortality, change in functional status should be considered as another relevant outcome in aging generations. However, there has been no community-based appraisal of functional outcomes between PG and TG. Using an administrative database, we compared functional outcomes between PG and TG. Among 12,508 patients who survived for $\geq 15$ years and underwent open gastrectomy between 2008 and 2010, we examined patient characteristics, comorbidities, functional status estimated by the Barthel index (BI) at admission and discharge, complications, ICU care, ventilation administration, blood transfusion, operating room time, resumption of oral intake, length of stay and total charges. With reference to distal gastrectomy (DG), we performed multivariate analyses to assess the impacts of PG and TG on complications and BI deterioration. A total of 434 PGs and 4,941 TGs were observed in 148 and 295 hospitals, respectively. Patient characteristics, care process, resumption of oral intake, operating room time, length of stay and total charges were also significantly different among the three gastrectomy types. PG, TG and DG
\end{abstract}

Kazuaki Kuwabara, MD, MPH, DPH Department of Health Care Administration and Management 
were not associated with complications or functional deterioration. Patient characteristics, preoperative blood transfusion and longer operating room time were significantly associated with more complications and BI deterioration. Since patient case mix and longer operating room time were associated with poor outcomes, physicians should recognize the role of PG and might optimally challenge and complete gastrectomies within the appropriate indications.

\section{Introduction}

Based on recent changes in the epidemiology of gastric cancer, particularly the fact that the incidence of upper stomach cancer has risen in Western countries, the clinical significance of open proximal gastrectomy (PG) has often been discussed through comparison of short- and long-term outcomes between open PG and open total gastrectomy (TG) [1-6]. Furthermore, based on recent demographic changes in the increasingly aged generation, surgical procedures would be indicated more for older patients with more comorbidities or disabled functional status.

Since the long-term survival outcomes for patients who have undergone PG have been observed to be equivalent to those for TG, PG has been gradually accepted as the optimal surgery for the upper stomach. It is also associated with a lower frequency of postoperative regurgitation and malnutrition, resulting in better postoperative health-related quality of life compared with TG $[2,3,5,6]$. However, studies on the variation in both functional outcomes and the traditional outcomes of complications and mortality are rare because detection of gastric carcinoma in its advanced stages is less common in Western countries than in the East Asian countries of Japan and South Korea [7]. Data on quality of care and life are likely to be based on single-center or randomized studies with a limited number of patients and to require a longer study period to obtain definitive conclusions when relatively more patients are involved [16]. In addition, those conclusions might need to be interpreted with caution because both laparoscopic gastrectomy and multidisciplinary care prevail during these longer periods and might result in variation in practice [8]. To address these varying circumstances surrounding PG, the use of a larger administrative database with information on functional outcomes, such as postoperative commencement of oral intake and changes in physical activity in daily life during hospitalization, would be promising for execution of a comparative quality study among PG, TG and distal gastrectomy (DG).

Using a Japanese administrative database including the Barthel index (BI) at admission and discharge and the date and quantity of dispense of medical care items, we aimed to compare functional outcomes at discharge between transabdominal PG and TG with reference to the outcomes of patients who underwent open DG. 


\section{Methods}

\section{Study Database}

Using the Japanese administrative database constructed by the Ministry of Health, Labour and Welfare (MHLW) to develop a case mix classification and determine the payment system, we analyzed patients who underwent PG, TG or DG without thoracotomy at hospitals participating in our case mix project. This database was originally developed by the MHLW as a Japanese case mix classification system in the fiscal year 2002 in conjunction with our research team. It helped establish hospital performance profiles and hospital payment systems in 1,525 hospitals (84 academic hospitals and 1,441 community hospitals) in 2010. The hospitals involved are responsible for delivering acute care, promoting medical research and educating students and postgraduate trainees. This national database comprises discharge summaries, claims data for each hospital and stores information on the quantity or date of every care item provided. It is collected annually between July 1 and December 31 .

Our original database included data on $8,177,721$ patients in 1,131 hospitals collected between 2008 and 2010. From this group, 17,909 patients from 303 hospitals underwent open gastrectomy (612 PGs across 166 hospitals, 6,970 TGs across 299 hospitals, and 10,327 DGs across 302 hospitals). The MHLW has managed to categorize the surgical procedure code for gastrectomy into three groups: PG, TG and DG, either through laparoscopy or laparotomy. We selected patients aged $\geq 15$ years who had survived at discharge across all hospitals participating in this project consecutively for the 3 years since 2008. We excluded patients with missing or unspecified BI at admission and discharge. Our research project was approved by the Ethics Committee of the University of Occupational and Environmental Health (Kitakyushu, Japan).

\section{Variable Definition}

Among the three gastrectomy types (PG, TG and DG), we examined age, sex, mortality, use of an ambulance, principal diagnosis at admission, presence of comorbidities, functional status as activities of daily life measured by BI at admission and discharge, discharge destination (at home or at other facilities), ICU care, ventilation, blood purification (hemodialysis, hemodiafiltration or hemadsorption), blood transfusions, presence of lymph node dissection during gastrectomy, administration of chemotherapy or radiation, complications, hospital patient volume, hospital teaching status, day of commencing postoperative oral intake of a semi-solid diet, operating room time (minutes), length of stay (days) and total charge (1 USD $=90 \mathrm{JPY})$.

Patients were divided into three age groups: $15-64,65-74$ and $\geq 75$ years. The diagnoses in the database were coded according to the International Statistical Classification of Diseases, 10th version (ICD10). The principal diagnoses were grouped into benign, primary and associated other malignancy. Primary was C16 and D002 in gastric cancer. Associated other malignancy was assigned an ICD code corresponding to malignant neoplasm except for C16 and D002. The remaining diagnoses were assigned ICD codes corresponding to benign neoplasm.

To evaluate the severities of chronic comorbid conditions, the Charlson comorbidity index (CCI) determined by ICD10 codes was used [9]. In addition, a maximum of four unexpected complications, defined as adverse events after admission, were recorded. Complications were summarized as either minor or major complications. Minor complications were assigned when the ICD codes corresponded to wound complications, hematoma, laceration or disruption of organs by instrumentation or manipulation (T81-T87) [10]. Major complications included reoperation for hemostasis, evacuation of hematoma or intra-abdominal abscess, enterostomy, fistulization or bowel resection after the study surgery and lysis of bowel obstruction.

In this study, functional outcomes were addressed by BI improvement during hospitalization and commencement of postoperative oral intake. The BI measures the ability of patients to perform 10 activities of daily life (feeding, grooming, bathing, dressing, bowel and bladder care, toilet use, ambulation, transfers and stair climbing) on a quantitative scale of five-point increments. The BI score ranged from 0 (a totally dependent bedridden state) to 100 (fully independent in physical functioning) [11]. BI at admission was categorized as dependent (BI <59), partially independent requiring assistance (BI 60-84) or nearly completely independent (BI $\geq 85$ ) [11]. Functional recovery was measured as the $\mathrm{BI}$ at discharge minus the $\mathrm{BI}$ at admission and was classified into one of three $\mathrm{BI}$ change categories: amelioration, no change or deterioration. 
The database contains the dates or amount of every medical case, and we were able to examine preoperative and postoperative ICU stays, ventilation, blood purification, blood transfusions and the postoperative day of commencing semi-solid food intake after gastrectomy. We were also able to calculate the amount ( $\mathrm{ml}$ ) of blood used for preoperative, postoperative and overall blood transfusions. Hospitals were classified as academic or community.

For total charges, charges for hospital care are determined by a standardized fee-for-service payment system of the national uniform tariff table and considered to be a good estimate of healthcare costs [12]. Total charges included fees for hospital administration and physicians as well as for instruments, laboratory studies and imaging.

Operating room time was defined as the time required for the anesthetist's procedures, preparation and positioning of video images and skin-to-skin time. Hospital volume was then averaged over 3 years and divided into three groups: low patient volume hospitals (182 hospitals, 1-40/year), medium volume hospitals (78 hospitals, 41-65/year) and high volume hospitals (43 hospitals, 66-183/year).

\section{Statistical Analysis}

Categorical variables were reported as the numbers and percentages of patients in each of the three gastrectomy groups and compared using the Pearson $\chi^{2}$ test. Continuous variables among the three gastrectomy types were compared using the Kruskal-Wallis test. Day of postoperative oral intake, operating room time, length of stay, total charges as well as preoperative, postoperative and overall blood transfusions were compared among the three gastrectomy types. A multiple logistic regression model was used to determine the effects of $\mathrm{PG}$ and $\mathrm{TG}$ on complications and $\mathrm{BI}$ deterioration. To allow for the ceiling effect inherent in the BI, BI at admission categories were included in the models. Statistical analyses were performed using IBM-SPSS 19.0 (SPSS Inc., Chicago, Ill., USA) with $\mathrm{p}<0.05$ considered to be statistically significant. All reported $\mathrm{p}$ values were two-tailed and the level of significance was set at $\mathrm{p}<0.05$.

\section{Results}

Of 12,508 cases of open gastrectomy, 434 patients were treated with PG in 148 hospitals (76 across 26 academic hospitals) and 4,941 patients were treated with TG across 295 hospitals (702 across 36 academic hospitals). The median patient ages differed significantly among the three gastrectomy types (PG 70 years vs. TG 69 years, both lower compared with DG), and the percentage of patients aged $\geq 75$ years who underwent TG (30.7\%) was lower compared with DG (35.6\%) and PG $(34.3 \%)$. The proportion of gender, use of an ambulance, principal diagnosis, CCI, BI category at admission, teaching status, hospital volume and complications varied significantly among gastrectomy types, whereas fiscal year did not. The BI change category and destination were not significantly different, although the day of postoperative oral intake was significantly deterred in TG. Variation in the proportion of patients who underwent ICU care, blood purification and transfusion was observed; however, the days on which these treatments occurred did not vary. Operating room time, length of stay and total charges were also significantly different among the three gastrectomy types (table 1).

Neither PG nor TG was significantly associated with complications or BI deterioration. Patients aged $\geq 75$ years, CCI, preoperative blood purification and transfusion, high volume hospital and advancing fiscal year were significantly associated with more complications. Patients aged $\geq 75$ years, dependent functional status, preoperative blood transfusion, academic hospital and advancing fiscal year were accompanied by BI deterioration, whereas complications and high volume 
hospital were not. Longer operating room time was significantly associated with more complications and BI deterioration (table 2).

\section{Discussion}

With the use of a Japanese administrative database, we compared short-term changes in functional outcome at discharge and postoperative oral intake between PG and TG with reference to DG. The three gastrectomy types were not associated with complications or functional deterioration at discharge, although TG deterred the day of commencing postoperative oral intake of semi-solid food. Longer operating room time was significantly associated with more complications and BI deterioration.

Several studies on the quality of PG and TG have recognized the safety and feasibility of PG based on single-center studies with longer study periods of 7 to approximately 25 years. This study, accumulating over 600 patients who underwent PG excluding laparoscopic PG during only 3 years, attempted to enroll a wide patient case mix expressed by process-based severity of ventilation proxy of respiratory failure, blood purification of renal failure, blood transfusion of preoperative anemia and administration of chemo-radiation because these processes would be expected to affect patients' activities of daily life. The positive relationship between the change in BI and the change in health-related quality of life was reported by Giles et al. [13]. Specifically, health-related quality of life was reportedly likely to be lowest 1-3 months after surgery and to reach a plateau thereafter. Longer length of stay of Japanese acute care hospitals has been acknowledged to be due to an acute care hospital definition that differs from that of Western hospitals by the Organization for Economic Co-operation and Development [14]. Turning around this circumstance may provide helpful information to estimate postoperative functional recovery. Because length of stay was 25.7, 27.2 and 30.5 days for patients who underwent DG, PG and TG, respectively, comments on the advantage of short-term quality of life of PG over TG may be justified. Neither PG nor TG were accompanied by complications or BI deterioration, though PG was likely to have shorter operating room time, length of stay and total charges compared with TG. When the gastric cancer is restricted to the upper stomach or limited to the area of the lesser curvature, PG might be advocated over TG because a longer operating room time was associated with more frequent complications in this study.

Gastric resection for gastric cancer is less frequent in Western countries than in Japan; there are more colorectal surgeries in Western countries [15]. Some reports, including this study, have indicated that complications or mortality from gastric cancer are lower in Japan than in Western countries $[1,5,15]$. TG was reportedly originally indicated for more advanced gastric cancer or wider tumor involvement in the lesser curvature, whereas some studies justified the performance of $\mathrm{PG}$ regardless of cancer stage [4]. This discrepancy in the propensity for PG or TG might be cancelled by the greater familiarity with gastrectomy in Japan, which would in turn endorse PG as the optimal surgical option for gastrectomy for various kinds of patient case mixes, including functional status. Japan is now facing a remarkable increase in the aging generation compared with Western developed countries; thus, PG may be challenged 
more frequently because there was no significant variation in the proportion of the three gastrectomies.

Some limitations in our study require comment. First, this study was observational, and the information was gathered from discharged patients during 6-month periods (July 1 to December 31) for 3 years starting in 2008. However, the MHLW gathered all hospitals participating in this case mix project and plans to extend the study period to 12 months starting in 2010. This will also improve the exhaustiveness of patients who have undergone PG and the generalizability of results derived from this kind of study. Second, some important clinical data, including the primary location of gastric cancer, body weight and cancer stage, were not included. The MHLW has also requested that hospitals include cancer stage, height and body weight in this administrative database since 2010. These accumulated clinical data would control the propensity for PG or $\mathrm{TG}$, and the results of functional outcomes would be more validated. Third, excluded patients were likely to be older and comorbid and cared for in high volume and academic hospitals more frequently than the included patients, which might necessitate more cautious interpretation of these results (see online supplementary table 1, www.karger.com/doi/10.1159/000339715).

In conclusion, we used a Japanese administrative database to compare the patient demographics, care process, resource use, complications, functional status at admission and discharge as well as resumption of postoperative oral intake among patients who underwent PG, TG and DG. None of these three procedures were associated with complications or functional deterioration at discharge. Longer operating room time and preoperative care processes as proxy of disease severity were significantly associated with more complications and BI deterioration. Since PG was likely to be associated with a shorter operating room time than TG, PG might be optimally challenged and completed within the appropriate indications. 
Table 1. Patient characteristics, care process, outcomes and resource use among the three types of gastrectomy

\begin{tabular}{|c|c|c|c|c|}
\hline & DG & $P G$ & $\mathrm{TG}$ & $\mathrm{p}$ \\
\hline Patients & 7,133 & 434 & 4,941 & \\
\hline Hospitals (community/academic) & $263 / 37$ & $122 / 26$ & $259 / 36$ & \\
\hline \multicolumn{5}{|l|}{ Age } \\
\hline Median & $71[16]$ & $70[16]$ & 69 [15] & $<0.001^{*}$ \\
\hline 65-74 years & $2,354(33.0)$ & $142(32.7)$ & $1,764(35.7)$ & $<0.001$ \\
\hline 75 years or older & $2,537(35.6)$ & $149(34.3)$ & $1,515(30.7)$ & \\
\hline Male gender & $4,893(68.6)$ & $327(75.3)$ & $3,632(73.5)$ & $<0.001$ \\
\hline Ambulance used & $218(3.1)$ & $4(0.9)$ & $142(2.9)$ & 0.036 \\
\hline \multicolumn{5}{|l|}{ Principal diagnosis at admission } \\
\hline Benign & $371(5.2)$ & $27(6.2)$ & $100(2.0)$ & $<0.001$ \\
\hline Primary & $6,610(92.7)$ & $400(92.2)$ & $4,783(96.8)$ & \\
\hline Associated other malignancy & $152(2.1)$ & $7(1.6)$ & $58(1.2)$ & \\
\hline \multicolumn{5}{|l|}{ Charlson comorbidity index } \\
\hline 1 & $1,648(23.1)$ & $113(26.0)$ & $986(20.0)$ & $<0.001$ \\
\hline 2 & $779(10.9)$ & $42(9.7)$ & $558(11.3)$ & \\
\hline 3 & $517(7.2)$ & $21(4.8)$ & $480(9.7)$ & \\
\hline 4 or more & $241(3.4)$ & $10(2.3)$ & $212(4.3)$ & \\
\hline \multicolumn{5}{|l|}{ BI at admission category } \\
\hline Dependent & $150(2.1)$ & $3(0.7)$ & $68(1.4)$ & 0.007 \\
\hline Assisted independent & $89(1.2)$ & $5(1.2)$ & $52(1.1)$ & \\
\hline Completely independent & $6,550(91.8)$ & $414(95.4)$ & $4,658(94.3)$ & \\
\hline \multicolumn{5}{|l|}{ Hospital patient volume } \\
\hline Low-volume hospital & $2,633(36.9)$ & $141(32.5)$ & $1,775(35.9)$ & $<0.001$ \\
\hline Middle-volume hospital & $2,279(32.0)$ & $138(31.8)$ & $1,745(35.3)$ & \\
\hline High-volume hospital & $2,221(31.1)$ & $155(35.7)$ & $1,421(28.8)$ & \\
\hline Academic teaching status & $848(11.9)$ & $76(17.5)$ & $702(14.2)$ & $<0.001$ \\
\hline \multicolumn{5}{|l|}{ Fiscal year } \\
\hline 2008 & $1,972(57.1)$ & $123(3.6)$ & $1,361(39.4)$ & 0.184 \\
\hline 2009 & $2,220(56.9)$ & $155(4.0)$ & $1,525(39.1)$ & \\
\hline 2010 & $2,941(57.1)$ & $156(3.0)$ & $2,055(39.9)$ & \\
\hline \multicolumn{5}{|l|}{ Complications } \\
\hline Minor complication & $900(12.6)$ & $44(10.1)$ & $684(13.8)$ & $<0.001$ \\
\hline Major complication & $98(1.4)$ & $8(1.8)$ & $111(2.2)$ & \\
\hline Abscess drainage & $63(0.9)$ & $2(0.5)$ & $75(1.5)$ & 0.002 \\
\hline Operative hemostasis & $14(0.2)$ & $1(0.2)$ & $11(0.2)$ & 0.947 \\
\hline Bowel resection & $4(0.1)$ & $0(0)$ & $9(0.2)$ & 0.085 \\
\hline Procedure for obstruction & $12(0.2)$ & $4(0.9)$ & $15(0.3)$ & 0.005 \\
\hline Ostomy & $9(0.1)$ & $1(0.2)$ & $7(0.1)$ & 0.841 \\
\hline \multicolumn{5}{|l|}{ BI change category } \\
\hline Deterioration & $178(2.5)$ & 7 (1.6) & $120(2.4)$ & 0.261 \\
\hline No change & $6,297(88.3)$ & $392(90.3)$ & $4,467(90.4)$ & \\
\hline Amelioration & $238(3.3)$ & $15(3.5)$ & $137(2.8)$ & \\
\hline BI change, median points & $0[0]$ & $0[0]$ & $0[0]$ & $0.141^{*}$ \\
\hline Median commencement of oral intake, days & $5[2]$ & $5[3]$ & $6[3]$ & $<0.001^{*}$ \\
\hline Destination at home & $6,828(95.7)$ & $412(94.9)$ & $4,707(95.3)$ & 0.407 \\
\hline \multicolumn{5}{|l|}{ ICU stay, median days } \\
\hline Overall & 1,735 (24.3), 1 [1] & 75 (17.3), 1 [0] & 1,365 (27.6), 1 [1] & $<0.001,0.254^{*}$ \\
\hline Preoperative & $70(1.0), 2[3.3]$ & $* *$ & $35(0.7), 2[2]$ & $0.040,0.492 *$ \\
\hline Postoperative & $1,715(24.0), 1[1]$ & 75 (17.3), $1[0]$ & $1,357(27.5), 1[1]$ & $<0.001,0.327^{*}$ \\
\hline \multicolumn{5}{|l|}{ Median ventilation, days } \\
\hline Overall & $217(3.0), 2[4]$ & $13(3.0), 1[3]$ & $176(3.6), 2[4.8]$ & $0.272,0.720^{*}$ \\
\hline Preoperative & $18(0.3), 2[5.3]$ & $* *$ & $4(0.1), 1[9]$ & $0.059,0.273^{*}$ \\
\hline Postoperative & $206(2.9), 2[4]$ & $13(3.0), 1[3]$ & $172(3.5), 2[4.8]$ & $0.181,0.676^{*}$ \\
\hline
\end{tabular}




\begin{tabular}{|c|c|c|c|c|}
\hline \multicolumn{5}{|l|}{ Median blood purification, days } \\
\hline Overall & $89(1.2), 11[10]$ & $10(2.3), 10.5[13.3]$ & $42(0.9), 9[10.3]$ & $0.008,0.461^{*}$ \\
\hline Preoperative & $71(1.0), 3[7]$ & $9(2.1), 3[4]$ & $30(0.6), 2.5[4.3]$ & $0.002,0.986^{*}$ \\
\hline Postoperative & $84(1.2), 59[1.5]$ & $10(2.3), 41.6[0.8]$ & $41(0.8), 33.4[1.3]$ & $0.008,0.332^{*}$ \\
\hline \multicolumn{5}{|l|}{ Median blood transfusion, $\mathrm{ml}$} \\
\hline Overall & 1,404 (19.7), 800 [800] & 57 (13.1), 800 [400] & 1,351 (27.3), 800 [800] & $<0.001,0.201^{*}$ \\
\hline Preoperative & $621(8.7), 800[600]$ & $21(4.8), 1,200[1,000]$ & 435 (8.8), $800[800]$ & $0.017,0.530^{*}$ \\
\hline Postoperative & $1,114(15.6), 800[800]$ & $44(10.1), 800[400]$ & $1,201(24.3), 800[800]$ & $<0.001,0.203^{*}$ \\
\hline Lymph node dissection & 6,771 (94.9) & $410(94.5)$ & $4,888(98.9)$ & $<0.001$ \\
\hline \multicolumn{5}{|l|}{ Chemo-radiation } \\
\hline Chemotherapy & $319(4.5)$ & $9(2.1)$ & $356(7.2)$ & $<0.001$ \\
\hline Radiation & $3(0.0)$ & $0(0.0)$ & $7(0.1)$ & \\
\hline Chemo-radiation & $4(0.1)$ & $0(0.0)$ & $4(0.1)$ & \\
\hline \multicolumn{5}{|l|}{ Resource use } \\
\hline Median operating room time, min & $271[108]$ & 304 [119.8] & 320 [112] & $<0.001 *$ \\
\hline Median length of hospital stay, days & $21[14]$ & $21[12]$ & $24[17]$ & $<0.001^{*}$ \\
\hline Median total charge, USD & $15,720.3[5,621.1]$ & $16,576.1[4,887]$ & $19,777.1[6,742.4]$ & $<0.001^{*}$ \\
\hline
\end{tabular}

Figures in parentheses are percentages, figures in brackets are interquartile ranges. BI = Barthel index. * Compared using Kruskal-Wallis test; other comparisons were made using $\chi^{2}$ test. ${ }^{* *}$ No case. 
Table 2. Factors associated with complications and BI deterioration

\begin{tabular}{|c|c|c|}
\hline & $\begin{array}{l}\text { Complications, } \\
\text { odds ratio }[95 \% \mathrm{CI}]\end{array}$ & $\begin{array}{l}\text { BI deterioration, } \\
\text { odds ratio }[95 \% \mathrm{CI}]\end{array}$ \\
\hline \multicolumn{3}{|l|}{ Age (for 15-64 years) } \\
\hline 65-74 years & 1.102 [0.973-1.248] & $2.236[1.470-3.400]$ \\
\hline 75 years or older & $1.207[1.064-1.370]$ & $5.711[3.881-8.403]$ \\
\hline Male & $1.225[1.091-1.376]$ & $1.044[0.802-1.361]$ \\
\hline Ambulance & $0.964[0.690-1.348]$ & $1.058[0.575-1.950]$ \\
\hline \multicolumn{3}{|l|}{ Principal diagnosis (for benign) } \\
\hline Primary & $0.995[0.686-1.442]$ & $0.382[0.213-0.683]$ \\
\hline Associated other malignancy & $1.324[0.827-2.119]$ & $0.332[0.121-0.913]$ \\
\hline \multicolumn{3}{|l|}{ Charlson comorbidity index } \\
\hline 1 & $1.125[0.991-1.277]$ & $0.933[0.687-1.266]$ \\
\hline 2 & 1.169 [0.994-1.376] & $1.093[0.762-1.566]$ \\
\hline 3 & $1.370[1.149-1.633]$ & $1.164[0.768-1.764]$ \\
\hline 4 or more & $1.399[1.092-1.791]$ & $1.169[0.700-1.950]$ \\
\hline \multicolumn{3}{|l|}{ Gastrectomy type (for DG) } \\
\hline $\mathrm{PG}$ & $0.960[0.480-1.922]$ & $0.615[0.119-3.167]$ \\
\hline $\mathrm{TG}$ & $0.761[0.564-1.027]$ & $0.724[0.333-1.575]$ \\
\hline Lymph node dissection & $0.807[0.408-1.594]$ & $1.454[0.312-6.785]$ \\
\hline \multicolumn{3}{|c|}{ BI at admission category (for completely independent) } \\
\hline Dependence at admission & $0.940[0.642-1.378]$ & $1.881[1.136-3.112]$ \\
\hline Assisted independence at admission & $0.624[0.372-1.047]$ & $4.257[2.677-6.768]$ \\
\hline Preoperative ICU stay & $0.560[0.283-1.109]$ & $1.101[0.403-3.008]$ \\
\hline Preoperative blood purification & $2.876[1.002-8.255]$ & $1.589[0.282-8.950]$ \\
\hline Preoperative blood transfusion & $1.772[1.127-2.786]$ & $2.769[1.306-5.873]$ \\
\hline Chemotherapy or radiation & $0.926[0.766-1.121]$ & $1.277[0.896-1.819]$ \\
\hline Chemotherapy & $1.141[0.920-1.415]$ & $1.330[0.789-2.243]$ \\
\hline Complications & $* *$ & $1.282[0.955-1.722]$ \\
\hline \multicolumn{3}{|l|}{ Teaching status (for community) } \\
\hline Academic & $0.739[0.629-0.869]$ & $1.414[1.028-1.945]$ \\
\hline \multicolumn{3}{|c|}{ Hospital patient volume (for low-volume hospital) } \\
\hline Middle-volume hospital & $0.971[0.858-1.099]$ & $0.944[0.705-1.263]$ \\
\hline High-volume hospital & $1.170[1.035-1.323]$ & $1.125[0.842-1.502]$ \\
\hline \multicolumn{3}{|l|}{ Fiscal year (for 2006) } \\
\hline 1 more year advanced & $1.237[1.160-1.318]$ & $3.359[2.677-4.216]$ \\
\hline \multicolumn{3}{|l|}{ Operating room time } \\
\hline 1 more minute & $1.002[1.001-1.002]$ & $1.001[1.000-1.003]$ \\
\hline Hosmer-Lemeshow goodness-of-fit & 0.099 & 0.006 \\
\hline
\end{tabular}

$\mathrm{BI}=$ Barthel index; $\mathrm{CI}=$ confidence interval. ${ }^{* *}$ Not enrolled in the model. 


\section{References}

1 Mariette C, Castel B, Toursel H, Fabre S, Balon JM, Triboulet JP: Surgical management of and long-term survival after adenocarcinoma of the cardia. Br J Surg 2002;89:1156-1163.

2 Kondoh Y, Ishii A, Ishizu K, Hanashi T, Okamoto Y, Morita M, Nabeshima K, Nakamura K, Soeda J, Ogoshi K, Makuuchi H: Esophagogastrostomy before proximal gastrectomy in patients with early gastric cancers in the upper third of the stomach. Tokai J Exp Clin Med 2006;31:146-149.

-3 Katsoulis IE, Robotis JF, Kouraklis G, Yannopoulos PA: What is the difference between proximal and total gastrectomy regarding postoperative bile reflux into the oesophagus? Dig Surg 2006;23:325-330.

4 Ooki A, Yamashita K, Kikuchi S, Sakuramoto S, Katada N, Hutawatari N, Watanabe M: Clinical significance of total gastrectomy for proximal gastric cancer. Anticancer Res 2008;28:2875-2883.

5 Kim EM, Jeong HY, Lee ES, Moon HS, Sung JK, Kim SH, Lee BS, Noh SM, Song KS, Shin KS, Cho JS: Comparison between proximal gastrectomy and total gastrectomy in early gastric cancer. Korean J Gastroenterol 2009;54:212-219.

-6 Katai H, Morita S, Saka M, Taniguchi H, Fukagawa T: Long-term outcome after proximal gastrectomy with jejunal interposition for suspected early cancer in the upper third of the stomach. Br J Surg 2010;97:558-562.

7 Deans C, Yeo MS, Soe MY, Shabbir A, Ti TK, So JB: Cancer of the gastric cardia is rising in incidence in an Asian population and is associated with adverse outcome. World J Surg 2011;35:617-624.

-8 Kuwabara K, Matsuda S, Ishikawa KB, Horiguchi H, Fujimori K: Association of operating time and gastrectomy with initiation of postoperative oral food intake. Dig Surg 2011;28:157-162.

$>9$ Sundararajan V, Henderson T, Perry C, Muggivan A, Quan H, Ghali WA: New ICD-10 version of the Charlson comorbidity index predicted in-hospital mortality. J Clin Epidemiol 2004;57:1288-1294.

10 Zhan C, Miller MR: Administrative data based patient safety research: a critical review. Qual Saf Health Care 2003;12(suppl 2):ii58-ii63.

-11 Kugler C, Altenhöner T, Lochner P, Ferbert A: Hessian Stroke Data Bank Study Group ASH: Does age influence early recovery from ischemic stroke? A study from the Hessian Stroke Data Bank. J Neurol 2003;250:676-681.

-12 Hayashida K, Imanaka Y, Otsubo T, Kuwabara K, Ishikawa KB, Fushimi K, Hashimoto H, Yasunaga H, Horiguchi H, Anan M, Fujimori K, Ikeda S, Matsuda S: Development and analysis of a nationwide cost database of acute-care hospitals in Japan. J Eval Clin Pract 2009;15:626-633.

13 Giles LC, Hawthorne G, Crotty M: Health-related quality of life among hospitalized older people awaiting residential aged care. Health Qual Life Outcomes 2009;7:71.

14 Organization for Economic Co-operation and Development (OECD): Health at a Glance - OECD Indicators 2005. Paris, OECD Publishing, 2005, p 56.

15 Bonenkamp JJ, Songun I, Hermans J, Sasako M, Welvaart K, Plukker JT, van Elk P, Obertop H, Gouma DJ, Taat CW: Randomised comparison of morbidity after D1 and D2 dissection for gastric cancer in 996 Dutch patients. Lancet 1995;25:745-748. 